\title{
FATORES MOTIVACIONAIS RELACIONADOS À PRÁTICA DE GINÁSTICA RÍTMICA ${ }^{1}$
}

\author{
Anderson Simas Frutuoso \\ Universidade Federal de Santa Catarina, Florianópolis, Santa Catarina, Brasil \\ Maria Helena Kraeski \\ Universidade do Estado de Santa Catarina, Florianópolis, Santa Catarina, Brasil \\ Ruy Jornada Krebs \\ Universidade do Estado de Santa Catarina, Florianópolis, Santa Catarina, Brasil
}

\begin{abstract}
Resumo
O estudo apresenta uma análise dos fatores motivacionais na prática de Ginástica Rítmica. Os sujeitos do estudo foram 34 integrantes de uma equipe de ginástica rítmica de Florianópolis (SC), todas do sexo feminino, com faixa etária de 10 a 20 anos. O instrumento utilizado foi a ESCALA DE MOTIVOS PARA PRÁTICA ESPORTIVA (EMPE), adaptação brasileira do PMQ, de Barroso e Krebs (2007). Os resultados indicaram os fatores Afiliação e Técnica como os mais importantes, e Status e Liberação de Energia como os menos importantes. Conclui-se que as estratégias motivacionais para este grupo devem fundamentar-se no fortalecimento das relações interpessoais existentes nele e na evolução do treinamento, e que a cobrança por resultados em competições deve ser estimulada de forma gradativa.

Palavras-chave: Ginástica. Motivação. Atletas.
\end{abstract}

\section{Introdução}

\begin{abstract}
A Ginástica Rítmica (GR) é uma modalidade que possui uma ca- racterística marcante quanto à beleza e à plasticidade em sua prática, pois alia a expressão corporal e manejo de aparelhos com a música, sem perder o caráter esportivo. Nedialkova, Soares e Barros (2006, p. 6) a definem como uma "síntese integrativa da arte e do desporto". Laffranchi (2001) atribui à GR características tanto artísticas quanto competitivas, no aspecto artístico, ficam evidentes o talento e a criatividade da modalidade pelo domínio corporal e sua harmonia de movimentos; no aspecto competitivo, enquanto modalidade essencialmente feminina, é necessário um alto nível de desenvolvimento de valências físicas, técnicas, táticas e psicológicas,
\end{abstract}

1-O presente trabalho não contou com apoio financeiro de nenhuma natureza para sua realização. 
objetivando a perfeição na execução dos movimentos corporais com os aparelhos.

Por ser uma modalidade de grande plasticidade, a avaliação da GR torna-se muito criteriosa, o que acarreta na incessante busca pela perfeição dos movimentos, tendo como conseqüência grandes cobranças durante o período de treinamento, além de alguns sacrifícios para atingir um nível ideal de preparação física. Segundo Lanaro Filho e Bohme (2001), a exigência por graus elevados de flexibilidade e a melhora de determinadas capacidades motoras fazem com que a GR tenha uma seleção esportiva adiantada, em relação a outras modalidades, por volta dos 6 aos 9 anos de idade, o que implica em uma precoce exposição da criança a responsabilidades.

No meio deste processo de treinamentos e competições algumas variáveis psicológicas tornam-se fundamentais para a continuidade da participação ou para a manutenção do desempenho esportivo (ROLHFS et. al. 2008), destas variáveis, a motivação seja talvez, a mais citada, por possuir estreita ligação com a atividade física, uma vez que o termo, oriundo do latim movere, significa mover, movimentar, e a atividade física, por sua vez, é conceituada por Caspersen, Powell e Christerson (1985), como o movimento corporal produzido pelo sistema músculos esquelético que resulta em gasto energético maior do que os níveis de repouso.

Estudos sobre motivação no esporte parecem convergir seus resultados ao apresentar fatores relacionados ao aprimoramento do desempenho técnico e habilidades esportivas entre os considerados mais importantes para a participação esportiva, fato este observado nos estudos de Gürbuz, Altýntas, e Asci, (2007), com adolescentes turcos praticantes de futebol; Barcelona e Sanfelice (2004), com atletas argentinos de voleibol; Barroso (2007), com atletas universitários de Santa Catarina; e de Paim (2001), com adolescentes praticantes de futebol. No estudo de Sit e Lindner (2006), com estudantes japoneses do ensino médio entre 14 a 20 anos que participaram de esportes por razões competitivas ou recreativas, o resultado geral também apresentou o desenvolvimento da técnica entre os mais importantes, assim como o resultado obtido com estudantes americanos no estudo de Yan e McCullagh (2004), comparando a motivação esportiva em diferentes culturas.

Entretanto, estudos tratando especificamente de GR parecem divergir dos estudos anteriormente citados, Vieira, Botti e Vieira (2005), 
analisando fatores motivadores e estressantes de atletas da seleção brasileira juvenil de GR, apontaram o gosto pela prática como fator motivador mais significante, já no estudo de Interdonato et. al. (2008), com atletas de GR evidenciou-se uma grande importância dada ao fator saúde pela maioria das atletas. Estas divergências podem ser fruto dos diferentes contextos de cada trabalho, de forma que ainda não há um consenso sobre o perfil motivacional de atletas de GR. Cabe ressaltar também, que ambos estudos utilizaram métodos diferentes para analisar e conhecer os fatores motivacionais.

O conhecimento dos fatores que levam uma pessoa a executar alguma tarefa pode ser de extrema utilidade para que se alcance o objetivo final, além de auxiliar no entendimento do nível de comprometimento e prazer que a realização da atividade traz ao indivíduo. Quando nos referimos à atividade física e esportes, esses motivos podem ganhar um valor vital para a realização e continuidade dos mesmos, segundo Yan e McCullagh (2004), esse conhecimento pode evidenciar as razões dos atletas em seus atos. Essa necessidade de conhecer os fatores que levam um grupo de meninas a despender parte de seus dias treinando e praticando uma modalidade de grande plasticidade, porém, extremamente criteriosa, norteia a iniciativa desse estudo. Tendo isso em vista, procuramos analisar quais os fatores que motivam a prática de ginástica rítmica, buscando saber quais são considerados mais importantes para as mesmas, além de comparar as diferentes categorias da equipe estudada.

\section{Método}

Este é um estudo de natureza descritiva com características de estudo de caso. O consentimento livre e esclarecido de participação dos sujeitos foi obtido junto às mesmas e aos pais e/ou responsáveis pelas mesmas, tendo o estudo sido aprovado pelo Comitê de Ética em Pesquisa envolvendo Seres Humanos da Universidade do Estado de Santa Catarina, sob número de aprovação $n^{\circ}$ 69/2008.

Participaram 34 integrantes da equipe de ginástica rítmica da Associação Desportiva do Instituto Estadual de Educação / Universidade do Estado de Santa Catarina - ADIEE/UDESC de Florianópolis, todas do sexo feminino, com faixa etária de 10 a 20 anos. A escolha da amostra se deu por conveniência, e para uma melhor compreensão foi necessário estabelecer uma estratificação na amostra, da qual resulta- 
ram 4 grupos: Grupo 1 (Infantil): 12 ginastas com idade média de $10,67(+0,98)$ anos com 2,31 $(+1,19)$ de prática. Esse grupo, por tratar de atletas mais jovens que já fazem parte da equipe, mas estão tendo sua iniciação desportiva, apresenta no seu treinamento um enfoque competitivo, participando de competições em níveis estaduais e brasileiro, porém com uma menor exigência quanto ao desempenho. Sua rotina de treinamento é 4 vezes na semana. Em um período de aproximadamente 14 horas por semana; Grupo 2 (Juvenil): 8 ginastas com idade média de $13,13(+0,99)$ anos e 5,88 $(+3,36)$ de tempo de prática. Este grupo pertence à categoria imediatamente abaixo da adulta, portanto já apresenta muito do seu treinamento voltado para o desempenho. Participa de competições em nível estadual e brasileiro, seu treinamento compreende 5 dias na semana, em períodos de 25 horas semanais; Grupo 3 (Adulto): 4 ginastas com idade média de 19,5 (+ $1,29)$ anos e 9,5 $(+2,08)$ de tempo de prática, nesse grupo encontramse ginastas da categoria adulta e com alto nível técnico, com um treinamento completamente voltado para o rendimento. Nesta categoria as atletas participam em competições em nível estadual, brasileiro e, algumas delas, em nível internacional também. A rotina de treinamento deste grupo totaliza 30 horas semanais, dividas em 6 dias; e Grupo 4 (Praticantes não atletas) : 10 praticantes de ginástica com idade média de 14,1 $(+2,08)$ anos e 1,82 $(+2,18)$ de tempo de prática. Esse grupo caracteriza-se por ser um grupo que apresenta treinamento não tão intenso e pouco voltado para o rendimento, sendo um grupo que, em uma hierarquia, estaria acima das escolinhas esportivas, mas abaixo da equipe, funcionando como uma pré-equipe, daí serem designadas como praticantes e não ginastas. Nesse grupo a exigência é menor, os treinamentos ocorrem em apenas um período, duas vezes na semana. Esse grupo também participa de competições estaduais, porém com uma intenção muito mais voltada à participação que à competição, devido ao menor nível técnico em que se encontram. Sua rotina de treinamento é de dois dias na semana, totalizando 4 horas.

Utilizou-se como instrumento de medida a ESCALA DE MOTIVOS PARA PRÁTICA ESPORTIVA (EMPE) (KREBS; BARROSO, 2008), a adaptação brasileira do Participation Motivation Questionnaire - o PMQ, desenvolvido nos EUA por Gill, Gross e Huddlestone (1983) - adaptação essa que foi validada no Brasil por Barroso (2007), sendo adaptada para determinar os motivos para a prática esportiva de atletas adultos jovens brasileiros. 
A escala compreende 33 itens, cada um apresentando uma escala de 0 a 10, valores referentes ao grau de importância dado pela respondente. Cada um dos 33 itens do questionário está agrupado em um dos 7 fatores motivacionais: Fator 1 (status); Fator 2 (condicionamento físico); Fator 3 (liberação de energia); Fator 4 (contexto); Fator 5 (técnica); Fator 6 (afiliação); Fator 7 (saúde). A coleta de dados foi realizada no período final do treinamento/aula, após o preenchimento da escala foram calculadas as médias e desvios-padrão de idade e de tempo de prática, bem como de cada fator motivacional. As médias obtidas dos fatores foram classificadas segundo recomendação do protocolo do instrumento (fator motivacional nada importante: valores entre 0,0 e 0,99 ; pouco importante: entre 1,0 e 3,99; importante: entre 4 e 6,99; muito importante: entre 7 e 9,99 e totalmente importante: 10). Para a estatística inferencial usou-se o programa SPSS 15.0 for Windows, no qual foi realizado o Teste ANOVA OneWay, Post Hoc de Scheffé $(p<0,05)$ para comparar os sete fatores entre os quatro grupos.

\section{Resultados}

Os resultados obtidos em cada grupo foram agrupados em tabela para melhor visualização dos valores correspondentes ao fator. 
DOI: $10.5216 /$ rpp.v16i4.20659

Tabela 1: Médias e desvios-padrão atribuídos aos fatores em cada grupo

\begin{tabular}{|c|c|c|c|c|c|c|c|c|}
\hline FATORES & $\begin{array}{l}\text { Grupo } 1 \\
\text { Infantil }\end{array}$ & & $\begin{array}{l}\text { Grupo } 2 \\
\text { Juvenil }\end{array}$ & $\begin{array}{l}\text { Grupo } 3 \\
\text { Adulto }\end{array}$ & & $\begin{array}{l}\text { Grupo } 4 \\
\text { Não atletas }\end{array}$ & $\begin{array}{l}\text { MÉDIA } \\
\text { GERAL }\end{array}$ & \\
\hline Status & $\begin{array}{l}8,18 \\
1,57\end{array}$ & \pm & $6,79 \pm 2,29$ & $\begin{array}{l}6,93 \\
2,19\end{array}$ & \pm & $4,89 \pm 2,48^{\circ}$ & $\begin{array}{l}6,69 \\
1,36\end{array}$ & \pm \\
\hline $\begin{array}{l}\text { Condicionamento } \\
\text { Físico }\end{array}$ & $\begin{array}{l}8,97 \\
1,00\end{array}$ & \pm & $\begin{array}{l}6,33 \\
2,10\end{array} \quad \pm$ & $\begin{array}{l}7,17 \\
2,20\end{array}$ & \pm & $8,17 \pm 1,30$ & $\begin{array}{l}7,66 \\
1,15\end{array}$ & \pm \\
\hline $\begin{array}{l}\text { Liberação } \\
\text { de Energia }\end{array}$ & $\begin{array}{l}8,42 \\
0,96\end{array}$ & \pm & $6,23 \pm 2,29$ & $\begin{array}{l}7,42 \\
1,21\end{array}$ & \pm & $5,78 \pm 1,80^{\circ}$ & $\begin{array}{l}6,96 \\
1,19\end{array}$ & \pm \\
\hline Contexto & $\begin{array}{l}9,13 \\
0,75\end{array}$ & \pm & $6,40 \pm 2,01^{\circ}$ & $\begin{array}{l}7,25 \\
2,70\end{array}$ & \pm & $\begin{array}{l}5,66 \\
2,07\end{array} \quad \pm$ & $\begin{array}{l}7,11 \\
1,50\end{array}$ & \pm \\
\hline Técnica & $\begin{array}{l}9,29 \\
0,93\end{array}$ & \pm & $8,75 \pm 1,43$ & $\begin{array}{l}7,81 \\
1,20\end{array}$ & \pm & $7,55 \pm 1,42$ & $\begin{array}{l}8,35 \\
0,81\end{array}$ & \pm \\
\hline Afiliação & $\begin{array}{l}9,53 \\
0,64\end{array}$ & \pm & $8,88 \pm 1,47$ & $\begin{array}{l}8,80 \\
0,71\end{array}$ & \pm & $7,42 \pm 2,03^{\circ}$ & $\begin{array}{l}8,66 \\
0,89\end{array}$ & \pm \\
\hline Saúde & $\begin{array}{l}9,58 \\
0,95\end{array}$ & \pm & $8,00 \pm 1,80$ & $\begin{array}{l}7,67 \\
2,92\end{array}$ & \pm & $7,73 \pm 2,51$ & $\begin{array}{l}8,25 \\
0,90\end{array}$ & \pm \\
\hline
\end{tabular}

Obs: $\left({ }^{*}\right) \mathrm{p}<0,05 ;(* *) \mathrm{p}<0,01 ;(* * *) \mathrm{p}<0,001$

O Teste Anova One Way encontrou diferenças significativas entre as médias obtidas pelos grupos em todos os fatores, exceto no fator saúde. O grupo adulto não apresentou diferença significativa em nenhum de seus fatores, fato não ocorrido nos demais grupos, os quais apresentaram diferenças significativas entre si. As médias referentes à liberação de energia e contexto dos grupos infantil e juvenil apresentaram diferenças, bem como as médias de status, liberação de energia, contexto, técnica e afiliação entre os grupos infantil e não atletas.

\section{Discussão}

Ao analisar os resultados, nota-se uma queda das médias em relação ao aumento da idade, independente do nível de treinamento em que se encontram as ginastas. Os números nos levam a crer que quanto mais velhas, mais seletivas e críticas se apresentam, provavelmente pela experiência e maturidade adquirida, dado também exposto por Pereira apud. Cid (2002), o qual mostra alterações na motivação em função da idade dos sujeitos e contrariando os dados do mesmo estu- 
do de Cid (2002), que não encontrou diferenças significativas nos motivos em função do ano de escolaridade de jovens portugueses.

Para uma análise mais completa dos resultados foi necessário ordená-los de forma decrescente em importância, cabe salientar que a classificação de importância atribuída aos fatores pelos grupos não apresentou grande divergência, sendo que todas as médias figuraram entre importante e muito importante. Desta forma, os resultados indicaram que os fatores mais importantes, no geral, são: Afiliação, técnica, saúde, contexto, liberação de energia, condicionamento físico (estes classificados como muito importantes), condicionamento físico e status (estes classificados como importantes). Especificamente, os resultados dos grupos apresentaram a seguinte ordem: Infantil: Saúde, afiliação, técnica, contexto, condicionamento físico, liberação de energia e status, sendo todos classificados como muito importantes; Juvenil: Afiliação, técnica, saúde, status, contexto, condicionamento físico e liberação de energia, tendo os três primeiros classificados como muito importantes e os quatro restantes como importantes; Adulto: Afiliação, técnica, saúde, energia, contexto, condicionamento físico e status, tendo apenas o fator status classificado como importante, os demais como muito importantes; e o Grupo de não atletas: Condicionamento Físico, Saúde, Técnica, Afiliação, sendo estes muito importantes e os restantes, Liberação de Energia, Contexto e Status, classificados como importantes. Estas classificações foram obtidas através do enquadramento das médias obtidas.

Nota-se, apesar de estatisticamente não significativa e não tão distintos nas classificações, uma diferença na importância hierárquica de fatores quanto aos grupos, em especial quando comparamos os fatores condicionamento físico e afiliação dos grupos infantil, juvenil e adulto, que fazem parte da equipe e tem uma rotina de treinamentos mais volumosa, com o grupo de não atletas, que tem apenas duas aulas na semana em um período de tempo muito menor. Nos grupos referentes à equipe, o fator condicionamento físico figura entre os três fatores menos motivantes, diferente do grupo de não atletas, que tem nesse fator sua maior motivação, Serpa (2003) afirma que o condicionamento físico e o sentimento de poder físico denotam característica do período púbere, fato que, neste grupo, parece fazer sentido pelo fato de sua média de idade estar em torno dos 14 anos. Em contraponto, no fator afiliação, a média geral o classifica como mais importante, o que também se apresenta nos grupos juvenil e adulto, no grupo infantil é o 
segundo da seqüência, porém no grupo de não atletas aparece apenas como quarto fator motivacional mais importante, o que nos leva a crer que os as ginastas juvenis e adultas motivam-se, acima de tudo, pelas amizades que possuem dentro da equipe, concordando com os achados de Balbinotti, Saldanha e Balbinotti (2009), que em seu estudo com atletas de basquetebol de ambos os sexos, também encontrou grande importância atribuída por atletas do sexo feminino a fatores sociais e de afiliação. Este fato tende a ser positivo, uma vez que, segundo Weiss e Smith (2002), o sentimento de afiliação e amizade no esporte leva a uma maior satisfação e manutenção na participação, tendo importante relação com a variável psicológica motivação, Serpa (2003), ressalta que a afiliação estimula a evolução pessoal de jovens atletas. Estas variações podem ser explicadas pela Teoria da Hierarquia das Necessidades (MASLOW, 1954 apud ATKINSON; HILGARD, 2002), que apresenta-se em forma de pirâmide, tendo 5 estágios, a necessidade no topo da pirâmide só pode ser satisfeita quando todos os estágios inferiores estiverem também satisfeitos. No primeiro estágio (base da pirâmide) estão as necessidades fisiológicas básicas; logo acima, estão as necessidades de segurança (estes dois primeiros figurando como necessidades primárias). Em seguida temos as necessidades de afiliação (um caráter mais social), seguida pela necessidade de estima, em auto reconhecimento das nossas capacidades e o reconhecimento de nossas capacidades pelos outros, e no topo da pirâmide as necessidades de auto-realização. Assim sendo, as diferenças citadas anteriormente, quando aliadas à teoria, levam a crer que o grupo de não atletas ainda não satisfez suas necessidades fisiológicas básicas, como condicionamento físico e saúde, portanto não sente tanta necessidade de afiliação e muito menos de status, que figura como a auto-realização da pirâmide. Os demais grupos parecem não apresentar uma ligação tão forte com essa teoria.

Nos grupos competitivos o fator status merece destaque, partindo do pressuposto que a amostra é uma equipe esportiva, e dessa forma, objetiva a obtenção de bons resultados em competições. Curiosamente, o fator figurou entre os quatro primeiros da ordem decrescente de importância apenas na categoria juvenil, fato este que pode ser resultado do período de vida em que as mesmas se encontram, com média de idade de 13,13 anos, estando na adolescência, período em que as mesmas buscam se auto afirmar (PIRES et. al., 2004). Desta forma, parece que a busca do status e de resultados expressivos na categoria 
juvenil tem relação com esta necessidade, embora, não possa ser explicado apenas pela mesma. As ginastas novatas e as adultas, aparentemente, não deram a mesma importância ao fator. Nas ginastas adultas isto pode ser resultado de seu amadurecimento como pessoas e atletas, uma vez que, por terem um tempo maior de prática do esporte e participação em diversas competições nacionais e internacionais, as mesmas já estão estabelecidas como atletas de alto nível técnico e, embora não descuidem do fator técnica, esta auto afirmação não lhes é mais característica. Cabe ressaltar que, como afirmam Krebs et al. (2011), os atributos da pessoa, suas relações interpessoais, os papéis e as atividades nas quais a mesma está engajada, além dos diferentes contextos no qual se inserem as atletas levam a alterações dos motivos relativos à prática esportiva.

Certas observações precisaram ser feitas ao compararmos o presente estudo com os demais, alguns autores utilizam uma forma diferente de apresentar os resultados obtidos, enquanto este estudo apresenta seus resultados em uma hierarquia de valores numéricos, alguns usam a porcentagem para definir a importância do fator, de forma que se usou o recurso do enquadramento na classificação quanto ao grau de importância. Outra questão é que são limitados, também, os estudos direcionados ao caráter psicológico da ginástica rítmica, de modo que as comparações foram todas com base em estudos de modalidades diferentes da mesma. $\mathrm{O}$ único estudo que analisava a motivação na GR, com uma metodologia semelhante à do presente estudo, utilizou um diferente instrumento, o Inventário de Motivação para a Prática Desportiva, de Gaya e Cardoso (1998), no entanto podemos ainda estabelecer uma relação entre os achados do presente estudo com o de Interdonato et al., (2008), em que 78,20\% das participantes $(\mathrm{n}=13)$ consideraram os itens de saúde muito importante, nos casos de Amizade e Lazer, 50,3\%, e na Competência Desportiva, 50\%. Portanto os motivos relacionados à saúde foram considerados mais importantes pela maioria, o que parece muito similar aos resultados do grupo de não atletas do presente estudo. Outro estudo, realizado por Cid (2002), utilizando o Questionário de Motivação para Atividades Desportivas - QMAD (adaptação do PMQ, por Serpa e Frias), ficou caracterizado que indivíduos jovens apresentam motivos semelhantes ao grupo de não atletas. Os motivos considerados mais importantes foram a questão de estar em forma e fazer ami- 
zades, já os de menor ocorrência foram os motivos relacionados a uma realização pessoal.

É importante levar em conta na comparação entre estudos que utilizaram a EMPE, QMAD ou PMQ que alguns itens existentes nestes três instrumentos estão abrigados em diferentes fatores em cada um deles, portanto, nas comparações alguns fatores podem divergir no nome, mas convergir nos motivos. O fator diversão do PMQ e QMAD teve, na EMPE, grande parte de seus itens abrigados no fator liberação de energia e outros itens que compunham originalmente duas categorias no PMQ, afiliação e atividade em grupo, foram agrupados no fator afiliação. Isso gerou uma diferença considerável, sobretudo nos motivos relacionados à diversão, devido ao novo arranjo feito na EMPE, a ausência do fator diversão que nos estudos de Freitas (2003), com diferentes grupos étnicos; Barroso, et al. (2007), pesquisando atletas universitários de Santa Catarina; Teques, (2006), com atletas de futebol do sexo masculino, com idades entre os 12 e os 8 anos; e no estudo de Rosolen, Voser e Hernandez (2006), com equipes universitárias de futsal feminino figuraram sempre entre os mais motivantes, no presente estudo figuraram entre os menos cotados. Já em relação ao arranjo do fator afiliação, nenhuma grande alteração foi notada, todos os estudos apresentaram, assim como o presente, altos graus de motivação por afiliação, exceto Rosolen, Voser e Hernandez (2006), que teve a afiliação como último fator motivante entre atletas femininas de futsal universitário.

Como já relatado, o desenvolvimento da técnica esportiva parece ser consenso entre os motivos mais importantes à prática esportiva (GÜRBUZ; ALTÝNTAS; ASCI, 2007; BARCELONA; SANFELICE 2004; BARROSO et al., 2007; PAIM, 2001; SIT; LINDNER, 2006; YAN; MCCULLAGH, 2004), fato também observado neste estudo, sendo que, mesmo no grupo não pertencente à equipe, o fator sempre esteve entre os três primeiros da ordem de importância, de forma que os dados apresentam semelhança com os estudos pesquisados, seguindo a tendência observada nos mesmos.

No estudo de Barcelona e Sanfelice (2004), utilizando o PMQ, foi investigada a motivação em equipes de vôlei argentinas com objetivos distintos (competição e recreativos), semelhante à situação encontrada entre os grupos da equipe e o grupo de não atletas do presente estudo, mostrou-se que no grupo argentino que treinava para competir os fatores apresentaram-se na seguinte ordem: desenvolvimento de habili- 
dades, condição física, excitação ou desafio, afiliação, liberar tensão e status. Já o grupo mais voltado para recreação mostrou diferenças apenas na ordem de desenvolvimento de habilidades, condição física, onde a condição física ficou em primeiro e desenvolver habilidades em segundo. Ao compararmos os achados, apesar da semelhança na divisão de grupos, os resultados foram distintos. Nos grupos voltados ao treinamento competitivo do presente estudo, o condicionamento físico aparece como um dos últimos fatores motivacionais na hierarquia de importância, já no estudo argentino, no grupo voltado ao treinamento competitivo o oposto foi percebido. Entretanto, a importância atribuída pelos participantes ao fator status em ambos estudos fora pouca, em relação aos demais fatores; quando comparamos os grupos com intenções participativas e recreativas de ambos estudos, os resultados foram bastante próximos.

\section{Considerações finais}

Por fim, pode-se notar que o perfil das ginastas desta equipe reflete em atletas que querem cada vez mais melhorar suas habilidades e se orgulham de fazer parte do grupo e da modalidade, desta forma, para manter as atletas cada vez mais motivadas em seus treinamentos devem ser criadas estratégias motivacionais que contemplem as relações interpessoais existentes nele e a evolução do treinamento, para que as atletas estejam em constante evolução. A importância de resultados expressivos em competições é um fator que, ao que parece, tende a se tornar mais importante na adolescência, o que reforça a idéia que exigência de resultados e títulos nas categorias iniciais do treinamento tendem a desestimular as ginastas.

Os resultados encontrados foram bastante próximos ao conhecimento prático (adquirido ao acompanhar o grupo durante o período da pesquisa), exceto pelo fator saúde, que apresentou níveis de importância mais altos que o esperado quando do inicio de presente estudo, o que demonstra que quem trabalha com GR deve ter atenção quanto à saúde, mesmo quando trabalhar com atletas, pois o descuido com a saúde das atletas poderá acarretar em uma diminuição da motivação das mesmas.

É importante que a presente pesquisa tenha sua população-alvo estendida em uma maior amostra para obter visão mais fidedigna do quanto tais motivos são importantes para diferentes ginastas, com di- 
ferentes idades e níveis técnicos, dessa forma podendo esboçar um perfil das atletas e oferecer maior suporte a todos os profissionais que trabalham com a ginástica rítmica, ao oferecer parâmetros para o entendimento do aspecto motivacional de suas atletas.

PRACTICE PARTICIPATION MOTIVATION RHYTHMIC GYMNASTICS

\begin{abstract}
The present aimed to investigate the more important participation motivation factors for girls that practice Rhythmic Gymnastics. The subjects of this study were 34 members of a rhythmic gymnastics team from Florianópolis (SC) ranging in age from 10 to 20 years old. The instrument used was the ESCALA DE MOTIVOS PARA PRÁTICA ESPORTIVA (EMPE), Brazilian crosscultural adaptation of PMQ by Barroso and Krebs (2007). The results indicated that the most important factors are: Affiliation, Skills, and the less important factors are Status and Energy Release. We conclude that the motivational strategies for this group should be based on interpersonal relations and it should be based on training evolution too and the pushing to reach good results in competitions should be stimulated in a gradual way.
\end{abstract}

Keywords: Gymnastics. Motivation. Athletes.

\title{
FACTORES MOTIVACIONALES QUE INCIDEN EN LA PRÁCTICA DE LA GIMNASIA RÍTMICA
}

\section{Resumen}

El estudio presenta un análisis de los factores motivacionales que inciden en la práctica de la gimnasia rítmica. Los sujetos de estudio fueron 34 miembros de un equipo de gimnasia rítmica en Florianópolis (SC), todas mujeres, con edades entre 10 y 20 años. El instrumento utilizado fue la ESCALA DE MOTIVOS PARA PRÁTICA ESPORTIVA (EMPE), la adaptación brasileña del PMQ, Barroso y Krebs (2007). Los resultados indican los factores tecnica deportiva y afiliación como el más importante, y el estatus y liberación de energía como el menos importante. Llegamos a la conclusión de que las estrategias para motivar este grupo debe basarse en el fortalecimiento de las relaciones interpersonales y la evolución de la formación deportiva, y que la cobranza por los resultados en las competiciones debería aumentar gradualmente.

Palabras clave: Gimnasia. Motivación. Atletas.

\section{Referências}

ATKINSON, R. L.; HILGARD, E. R. Introdução à psicologia de Hilgard. 13. ed. Porto Alegre: Artmed, 2002. 790 p.

BALBINOTTI, M. A. A; SALDANHA, R. P.; BALBINOTTI, C. A. A. Dimensões motivacionais de basquetebolistas infanto-juvenis: um 
estudo segundo o sexo. Motriz: revista de educação física, v. 15, n. 2, p. 318-329, abr./jun. 2009. Disponível em: <http://www.periodicos.rc.biblioteca.unesp.br/index.php/motriz/article/view/2513/2356>. Acesso em: 23 dez. 2010.

BARCELONA, E. M.; SANFELICE, G. R. Motivos de atletas "riocuartenses" para jogar voleibol. Revista Digital, v.10, n.78, 2004. Disponível em <http://www.cbdu.org.br/?pg=sobre $>$. Acesso em 11 de ago. de 2008.

BARROSO, M. L. C. et al. Motivos de prática de esportes coletivos universitários em Santa Catarina. In: FÓRUM INTERNACIONAL DE ESPORTES, 6., 2007, Florianópolis. Anais... Florianópolis: FESPORTE, 2007. Disponível em: < http://www.unesporte.org.br/forum2007/apresentacao_oral/11_mario_luiz_barroso.pdf> Acesso em: 30 nov. 2010

BARROSO, M. L. C. Validação do Participation Motivation Questionnaire Adaptado para Determinar Motivos de Prática Esportiva de Adultos Jovens Brasileiros. Florianópolis: UDESC, 2007. 130 p.

CASPERSEN, C. J., POWELL, K. E., CHRISTERSON, G. M. Physical Activity, Exercise and Physical Fitness: Definitions and Distinctions for Health-Related Research. Public Health Reports. v. 100, n. 2, p.126-131, 1985.

CID, L. F. Alteração dos motivos para a prática desportiva das crianças e jovens. Revista Digital EF y Deportes, Buenos Aires, v.8, n.55, dez., 2002. Não paginado.

FREITAS, C. M. S. M. Satisfação com a imagem corporal e motivação para a prática desportiva em adolescentes escolares. Comparação dos três grupos Étnicos numa perspectiva Biopsicossociocultural. In: ENCONTRO PERNAMBUCANO DE PESQUISA EM EDUCAÇÃO FÍSICA E ESPORTE, 12, 2003, Recife. Anais... Recife: [s. n.], 2003.

GAYA, A.; CARDOSO, M. Os fatores motivacionais para a prática desportiva e suas relações com o sexo, idade e níveis de desempenho desportivo. Revista Perfil, Porto Alegre, v.2, n.2, p.40-51, 1998. 
GILL, D. L.; GROSS, J. B.; HUDDLESTON, S. Participation motivation in youth sports. International Journal of Sports Psychology, n.14, p.1-14, 1983.

GÜRBUZ, B.; ALTÝNTAS, A.; ASCI, F. H. A. Participation Motives of 9-15 Years Old Turkish Soccer Players. Journal of Sports Science and Medicine, Antalya, v. 6, s.10, p.88-90, fev., 2007.

INTERDONATO, G. C. et al. Fatores Motivacionais de Atletas Para a Prática Esportiva. Motriz, Rio Claro, v.14 n.1 p.63-66, jan./mar. 2008.

KREBS, R. J., BARROSO, M. L. C. EMPE: Brazilian Young Adults Sports Participation Motivation Assessment In: ANNUAL CONGRESS OF THE EUROPEAN COLLEGE OF SPORT SCIENCE, 13., 2008, Estoril (Portugal). Anais... Lisboa: Faculdade de Motricidade Humana, 2008. v. 1. p.612-613.

KREBS, R. J. et al. Disposição de adolescentes para a prática de esportes: um estudo orientado pela Teoria Bioecológica de Bronfenbrenner. Motriz, v.17 n.1, p.195-201, jan./mar., 2011. Disponível em: $<\mathrm{http}$ ://www.periodicos.rc.biblioteca.unesp.br/index.php/motriz/article/view/1980-6574.2011v17n1p195/pdf>. Acesso em: 23 mar. 2011.

LAFFRANCHI, B. Treinamento desportivo aplicado à ginástica rítmica. Londrina: UNOPAR, 2001. 157 p.

LANARO FILHO, P., BÖHME, M. T. S. Detecção, Seleção E Promoção De Talentos Esportivos Em Ginástica Rítmica Desportiva: Um Estudo De Revisão. Revista paulista de Educação Física, São Paulo, v. 15 , n. 2 , p. 154-68, jul./dez., 2001

NEDIALKOVA, G.; SOARES, A. A.; BARROS D. Ginástica Rítmica: Em busca de novos talentos. Petrópolis: Portal Literário, 2006. 94 p.

PAIM, M. C. C. Fatores Motivacionais e Desempenho no Futebol. Revista da Educação Física/UEM Maringá, v. 12, n. 2, p. 73-79, jul./dez., 2001.

PIRES, E. A. G. et al. Hábitos de atividade física e o estresse em adolescentes de Florianópolis-SC, Brasil. Revista brasileira de Ciência e Movimento, Taguatinga, DF, v. 12, n. 1, p. 51-56, 2004 
ROHLFS, I. C. P. M. et al. Escala de Humor de Brunel (BRUMS): Instrumento para detecção precoce da síndrome do excesso de treinamento. Revista Brasileira de Medicina do Esporte, Niterói, v. 14, n. 3, p.176-181, maio/jun., 2008.

ROSOLEN, M.; VOSER, R. C.; HERNANDEZ, J. A. E. A motivação para o esporte em atletas universitárias de futsal feminino. Futsal Brasil: O Portal do Futsal Mundial, 2006. Disponível em $<$ http://www.futsalbrasil.com.br/artigos/artigo.php?cd_artigo=143>. Acesso em 22 de ago. de 2008.

SAMULSKI, D. Psicologia do Esporte. São Paulo: Manole, 2002

SERPA, S. Treinar Jovens: Complexidade, Exigência E Responsabilidade Revista da Educação Física/UEM, Maringá v. 14, n. 1, p. 7582, jan./jun., 2003.

SIT, C. H. P.; LINDNER, K. J. Situational state balances and participation motivation in youth sport: A reversal theory perspective. British Journal of Educational Psychology, n. 76, p. 369-384. 2006

TEQUES, P. H. A. Motivos e orientações cognitivas para a prática do futebol juvenil de competição. In: CONGRESO DE LA SOCIEDAD IBEROAMERICANO DE PSICOLOGÍA DEL DEPORTE. 2006. Anais... [S. 1.: s. n.], 2006. p. 12-16.

VIEIRA, L. F.; BOTTI, M. VIEIRA, J. L. L. Ginástica rítmica - análise dos fatores competitivos motivadores e estressantes da Seleção Brasileira Juvenil. Acta Scientiarum Health Science, Maringá, v. 27, n. 2 , p. $207-215,2005$

WEISS, M. R., SMITH, A. L. Friendship Quality in Youth Sport: Relationship to Age, Gender, and Motivation Variables. Journal of Sport \& Exercise Psychology, n. 24, p. 420-437. 2002

YAN, J. H., McCULLAGH, P. Cultural Influence on Youth's Motivation of Participation in Physical Activity. Journal of Sport Behavior, v. 27, n. 4,2004 .

Recebido em: 16/10/2012

Revisado em: 10/01/2013

Aprovado em: 18/02/2013

Pensar a Prática, Goiânia, v. 16, n. 4, p. 956-1270, out./dez. 2013 
DOI: $10.5216 /$ rpp.v16i4.20659

\section{Endereço para correspondência}

maria.kraeski@udesc.br

Maria Helena Kraeski

Universidade do Estado de Santa Catarina, Centro de Ciências da Saúde e do Esporte.

Rua Paschoal Simone 358

Coqueiros 